\title{
SINR Profile for Spectral Efficiency Optimization of SIC Receivers in the Many-User Regime
}

\author{
Josep Sala-Alvarez, Javier Villares and Francesc Rey \\ Dept. of Signal Theory and Communications, Universitat Politècnica de Catalunya - Barcelona Tech \\ c/ Jordi Girona 1-3, Campus Nord UPC, Edifici D5, 08034 Barcelona (Spain) \\ Email: \{josep.sala,javier.villares,francesc.rey\}@upc.edu
}

\begin{abstract}
In dense wireless scenarios, and particularly under high traffic loads, the design of efficient random access protocols is necessary. Some candidate solutions are based on DirectSequence Spread Spectrum (DS-SS) combined with a Successive Interference Cancellation (SIC) demodulator, but the performance of these techniques is highly related to the distribution of the users received power. In that context, this paper presents a theoretical analysis to calculate the optimum user SINR profile at the decoder maximizing the spectral efficiency in $\mathrm{bps} / \mathrm{Hz}$ for a specific modulation and practical Forward Error Correction (FEC) code. This solution is achieved by means of Variational Calculus operating in the asymptotic large-user case. Although a constant SINR function has been typically assumed in the literature (the one maximizing capacity), the theoretical results evidence that the optimum SINR profile must be an increasing function of the users received power. Its performance is compared with that of the uniform profile for two representative scenarios with different channel codes in a slightly overloaded system. The numerical results show that the optimum solution regulates the network load preventing the aggregate throughput from collapsing when the system is overloaded. In scenarios with a large number of transmitters, this optimum solution can be implemented in an uncoordinated manner with the knowledge of a few public system parameters.
\end{abstract}

Keywords-Spread Spectrum, Multiple-Access, Successive Interference Cancellation, Spectral Efficiency, Throughput, Variational Calculus, Uncoordinated Access

\section{INTRODUCTION}

The random (or uncoordinated) multiple access problem constitutes a major challenge in future massive wireless networks [1]. In such novel scenarios, collisions can be potentially very critical, specially when the number of terminals grows unboundedly. A common feature of recent uncoordinated multiple-access protocols is that they tackle collisions, not as a drawback, but as an opportunity to increase the throughput. In most cases, this benefit is reaped by introducing a Successive Interference Cancelation (SIC) scheme that mitigates the interference produced by collisions [2]-[5]. The performance of some SIC-based random access solutions is highly dependent on the received power distribution [6] and benefits from power unbalance, as is the case in Enhanced Spread Spectrum Aloha (E-SSA) schemes [3]. Therefore, we focus on the performance of the random access scheme in terms of the user power distribution.

This paper provides the analytical solution to the optimum symbol energy profile at the input of the SIC Direct-Sequence Spread Spectrum (DS-SS) receiver maximizing the system spectral efficiency in $\mathrm{bps} / \mathrm{Hz}$, for a specific FEC code (characterized by its Packet Error Rate curve). A general belief in the literature is that the optimum received signal to interference plus noise ratio (SINR) profile in a multiple-access DS-SS system is constant over successive SIC stages for all the users [8]. While this statement is true when maximizing the channel capacity [9] [10], no proof is available that a constant SINR profile is optimum in terms of spectral efficiency (throughput) when using specific and implementable modulation and coding schemes. In [11], trying to implement an admission control procedure, the authors derive the optimum received signalpower distribution which maximizes throughput, but omit the FEC (uncoded BER curves are assumed) and enforce, heuristically, a constant SINR for all transmitted packets. In this paper, we prove, by means of Variational Calculus, that in the asymptotic many-user case the optimum SINR profile must be an increasing function of the users received power when SIC and practical modulation and coding schemes are implemented. This asymptotic model and its optimization problem, both presented in Sec. II, match the potentially large population scenarios envisaged for future wireless networks. Sec. III and appendices present the related asymptotic analyses deriving the theoretical results for the per-user optimum $E_{s} / N_{0}$ and SINR profiles. In Sec. IV, numerical results corroborate, for two specific FECs, that the optimum SINR profile is not uniform, but rather, a decreasing function of the SIC decoding order. A more detailed examination of the results shows, that in overloaded systems, the optimum solution regulates the load avoiding the throughput drop-off due to an excess of collisions. The last part of the paper discusses, briefly, how the optimum solution could be implemented uncoordinatedly, a sensible approach when a large number of terminals perform random access.

\section{Problem StATEMENT}

Let $K$ asynchronous DS-SS users transmit to a common receiver and let $\gamma_{k}$ be the received $E_{s} / N_{0}$ of the $k$-th user, under the average received $E_{s} / N_{0}$ constraint $\bar{\gamma}_{K}$,

$$
\bar{\gamma}_{K}=\frac{1}{K} \sum_{k=1}^{K} \gamma_{k}
$$

We assume a successive interference cancellation strategy where users are decoded sequentially from the most powerful ( $k=K)$ to the least powerful $(k=1)$ one and where, each time a user is successfully decoded, a residual interference fraction $0<\varepsilon \leq 1$ of the current user's power remains due 
to imperfect cancellation [6]. After cancellation of previous users, the $k$-th user SINR is given by,

$$
\Gamma_{k}=\frac{\gamma_{k}}{1+\frac{\theta}{N} \sum_{i=1}^{k-1} \gamma_{i}+\varepsilon \frac{\theta}{N} \sum_{i=k+1}^{K} \gamma_{i}}
$$

with $\frac{\theta}{N}$ the average decorrelation factor assumed between signatures of different users in the long code model (spreading code much longer than the symbol period), $N$ the spreading factor and $0<\theta \leq 1$ an additional average decorrelation factor associated with random inter-user chip timing and/or carrier phase differences. The $k$-th user's Packet Error Rate (PER), or probability of decoding incorrectly the $k$-th user packet, is a function of $\Gamma_{k}$ depending on the adopted modulation and channel-coding scheme: $\operatorname{per}_{k}=\operatorname{PER}\left(\Gamma_{k}\right)$. For a SIC policy whereby decoding is stopped when a packet decoding error occurs, the system spectral efficiency in bps/Hz becomes,

$$
E_{K}=\frac{R_{c}}{N} \sum_{k=1}^{K} \prod_{i=k}^{K}\left(1-\operatorname{per}_{i}\right)
$$

with $R_{c}$ the number of information bits per channel use of the adopted modulation and coding scheme. In this context, our objective is to derive the optimum $E_{s} / N_{0}$ profile $\left\{\gamma_{k}\right\}_{k=1, \ldots, K}$ and its associated SINR profile $\left\{\Gamma_{k}\right\}_{k=1, \ldots, K}$ that maximize $E_{K}$ (we may refer to either one of them interchangeably during the optimization). In the finite-user case ( $k$ discrete), this problem cannot be solved analytically. Alternatively, as in [9] [10], we evaluate the asymptotic case in which $K, N \rightarrow \infty$ while the traffic load $\alpha=K / N$ remains constant. This will be useful in obtaining tight approximations when $K, N>>1$.

One may be interested in the effect of using the asymptotic $E_{s} / N_{0}$ profile when the number of users is finite. We refer the reader to the analysis in [7], which, although implementing a different SIC policy, provides a detailed comparison of average PER results between the user-asymptotic and finite-user cases. A good agreement is observed therein at $K=512$ users.

\section{A. User-asymptotic expressions}

In the asymptotic case, the discrete index $k$ is replaced with the continuous index $t \doteq \lim _{K \rightarrow \infty} k / K(0<t \leq 1)$, and the problem can be reformulated in terms of the (normalized) continuous $E_{s} / N_{0}$ profile, $\dot{y}(t)$, and the (normalized) aggregate $E_{s} / N_{0}$ profile, $y(t)$, defined as

$$
\begin{aligned}
\dot{y}(t) & \doteq \frac{1}{\bar{\gamma}} \lim _{K \rightarrow \infty} \gamma_{t K} \\
y(t) & \doteq \frac{1}{\bar{\gamma}} \lim _{K \rightarrow \infty} \frac{1}{K} \sum_{i=1}^{t K-1} \gamma_{i}=\int_{0}^{t} \dot{y}(u) \mathrm{d} u
\end{aligned}
$$

where the normalization constant $\bar{\gamma} \doteq \lim _{K \rightarrow \infty} \bar{\gamma}_{K}$ is the asymptotic average received $E_{s} / N_{0}(1)$, the dot accent denotes differentiation in $t$, i.e., $\dot{y}(t)=\nabla_{t} y(t) \doteq \frac{\mathrm{d}}{\mathrm{d} t} y(t)$ and where the differential $\mathrm{d} u$ is obtained from the asymptotic equivalence $\mathrm{d} u \equiv 1 / K$ when $K \rightarrow \infty$.

From the above definitions, we can obtain the equivalent asymptotic values of $\Gamma_{k}$ in (2) and $E_{K}$ in (3). Firstly, we substitute (4) and (5) into (2) and we get,

$$
\Gamma(t) \doteq \lim _{K \rightarrow \infty} \Gamma_{k}=\frac{\dot{y}(t)}{a+b y(t)}=\frac{1}{b} \nabla_{t} \ln (a+b y(t))
$$

with parameters $a, b$ easily defined from (2) and $\alpha=\frac{K}{N}$ as,

$$
a \doteq(1+\alpha \theta \varepsilon \bar{\gamma}) / \bar{\gamma} \quad, \quad b \doteq(1-\varepsilon) \alpha \theta
$$

Thus, equation (6) determines the asymptotic SINR profile $\Gamma(t)$ from the asymptotic normalized $E_{s} / N_{0}$ profile $y(t)$. The determination of $y(t)$ from $\Gamma(t)$ is addressed in section III.

Secondly, by expressing the finite product $\Pi_{i=k}^{K}[\cdot]$ in (3) as $\exp \left(\sum_{i=k}^{K} \ln [\cdot]\right)$ and then letting the finite summation $\sum_{i=k}^{K} \ln [\cdot]$ in the exponent converge to an integral as done in (5), we get the following tight approximation $E$ to the system spectral efficiency $E_{K}$ in (3) for a sufficiently large number of users $K=\alpha N$,

$$
E_{K} \rightarrow E \doteq \alpha R_{c} \int_{0}^{1} \exp \left(\alpha N \int_{u}^{1} f(\Gamma(v)) \mathrm{d} v\right) \mathrm{d} u(8)
$$

where we have used that each stage's PER converges as per $_{k=t K} \rightarrow \operatorname{PER}[\Gamma(t)]$, and where we have also defined,

$$
f(\Gamma) \doteq \ln (1-\operatorname{PER}(\Gamma))<0
$$

a negative function over $\Gamma$ of the adopted modulation/coding PER characteristic: the logarithm of the Packet Success Rate $\operatorname{PSR}[\Gamma] \doteq 1-\operatorname{PER}[\Gamma]$. Note that, as will be used, $f(\Gamma)$ increases with $\Gamma$, with the following positive derivative in $\Gamma$,

$$
f^{\prime}(\Gamma)=\frac{\operatorname{PSR}^{\prime}[\Gamma]}{\operatorname{PSR}[\Gamma]}>0
$$

as any practical function $\operatorname{PSR}[\Gamma]$ should increase with $\Gamma$. Moreover, as $\lim _{\Gamma \rightarrow \infty} \operatorname{PSR}[\Gamma] \rightarrow 1^{-}$, we conclude that $f^{\prime}(\Gamma)$ decreases for $\Gamma \geq \Gamma_{f}$, with $\Gamma_{f}$ specific to the modulation/coding scheme.

\section{B. Optimization problem}

The optimization problem consists in maximizing the functional $E$ in (8) with respect to $y(t)$ in (5) under the boundary constraints $y(0)=0$ and $y(1)=1$, where the former is trivially satisfied and the latter fixes the average received $E_{s} / N_{0}$ (1). As in [9, Eq. 25], this is a variational calculus problem that is solved analytically in Sec. III from the modified Euler-Lagrange equation derived in Appendix I, which proves that a necessary condition for the profile $\Gamma(t)$ (corresponding to $y(t))$ to maximize $E$ is that the following invariance equation be fulfiled for any $t$,

$$
\mathrm{E}(t) \cdot f^{\prime}(\Gamma(t))=c_{0}
$$

where the term $\mathrm{E}(t)$, defined from $\Gamma(t)$ as follows,

$$
\mathrm{E}(t) \doteq \alpha R_{c} \int_{0}^{t} \exp \left(\alpha N \int_{u}^{1} f(\Gamma(v)) \mathrm{d} v\right) \mathrm{d} u
$$

constitutes the partial aggregate asymptotic spectral efficiency of users with indices $0<u<t$ and where each different value for the constant $c_{0}$ in (11) is associated with a different solution $y(t)$ to (11). We let that be denoted the solution space $y\left(t ; c_{0}\right)$. Therefore, we will select that solution $y(t)=y\left(t ; c_{0}\right)$, associated with a given $c_{0}$, that satisfies the average received $E_{s} / N_{0}$ constraint $\bar{\gamma}$. The following lemma sheds some light on the behaviour of the corresponding optimum SINR profile $\Gamma(t)$, whose expression is analytically obtained in Sec. III.

Lemma 1: The SINR profile $\Gamma(t)$ fulfiling the invariance equation (11) is an increasing function of $t$. 
Proof: Note that, by its definition in (12), E $(t)$ is increasing in $t$. Hence, from the invariance $(11), f^{\prime}(\Gamma(t))$ must be decreasing in $t$. In consequence, as discussed after (10), the derivative $f^{\prime}(\Gamma)$ is a positive decreasing function in $\Gamma$, we conclude that $\Gamma(t)$ must be an increasing function of $t \diamond$.

The increasing character of the optimum SINR profile $\Gamma(t)$ in $t$ stated by Lemma 1 establishes that efficiency is maximized when the SINR of the first decoded users is prioritized. This differs radically from the results of capacity studies where the optimum SINR profile is found to be uniform [9]. The reason is that we are considering a practical modulation and coding scheme whereas a capacity-achieving error-free modulation/coding is implicitly used in asymptotic capacity studies. Our goal in the following sections is thus to obtain an analytical expression for the increasing optimum profile $\Gamma(t)$ and to compare its performance with that of the capacitymaximizing uniform profile in [9].

\section{OPTIMUM SINR AND $E_{s} / N_{0}$ PROFILES}

Firstly, rather than finding the solution $y(t)$ to the invariance equation (11), we consider its corresponding solution $\Gamma(t)$. The optimum SINR profile $\Gamma(t)$ can be obtained by solving the nonlinear integro-differential equation jointly determined by (11)-(12). This step is, mathematically, rather involved and is documented in appendix II. Its solution space, denoted $\Gamma_{\mathrm{opt}}(t ; c)$ (see appendix II), depends on a constant $c$ which may also be determined from the average $E_{s} / N_{0}$ constraint $\bar{\gamma}$. Note that here we are considering a parameter $c$ for the solution space $\Gamma_{\mathrm{opt}}(t ; c)$, which is different from its corresponding parameter $c_{0}$ for the solution space $y\left(t ; c_{0}\right)$. In fact, both $c$ and $c_{0}$ will be determined from the values of $a, b$ in (7), which, in their turn, depend on $\bar{\gamma}$.

Secondly, we determine the corresponding $y(t)$ and $\gamma(t) \doteq$ $\bar{\gamma} \dot{y}(t)$ from $\Gamma(t)$. Note now that the boundary condition $y(1)=$ 1 can be cast in terms of $\Gamma(t)$ : if $y(t)$ is evaluated from (6) using $y(0)=0$, we obtain

$$
y(t)=\frac{a}{b} \exp \left(b \int_{0}^{t} \Gamma(u) \mathrm{d} u\right)-\frac{a}{b}
$$

Then, substituting $y(1)=1$ into the equation above, the following equivalent constraint on the area of $\Gamma(t)=\Gamma_{\mathrm{opt}}(t ; c)$ results, which is associated with the corresponding parameter $c$ of the solution space $\Gamma_{\mathrm{opt}}(t ; c)$,

$$
\int_{0}^{1} \Gamma_{\mathrm{opt}}(u ; c) \mathrm{d} u=\frac{1}{b} \ln \left(1+\frac{b}{a}\right) \doteq \bar{\Gamma}_{0}
$$

where $\bar{\Gamma}_{0}$ (depending on $\bar{\gamma}$ through $a, b$ ) corresponds to the SINR of the constant profile maximizing capacity [9]. Once the optimal SINR profile is found, the optimum continuous $E_{s} / N_{0}$ profile can be computed from the derivative of (13) evaluated at $\Gamma(t)=\Gamma_{\mathrm{opt}}(t ; c)$, yielding

$$
\begin{aligned}
\gamma_{\mathrm{opt}}(t) & =\left.\bar{\gamma} \dot{y}(t)\right|_{\Gamma_{\mathrm{opt}}} \\
& =a \bar{\gamma} \Gamma_{\mathrm{opt}}(t) \exp \left(b \int_{0}^{t} \Gamma_{\mathrm{opt}}(u) \mathrm{d} u\right)
\end{aligned}
$$

\section{Numerical ANALYSiS}

The theoretical results derived in the previous sections are numerically evaluated in this section for a representative scenario with a spreading factor $N=2048$, a SIC residual interference power coefficient $\varepsilon=0.1$ and QPSK modulation. Assuming an interference dominated scenario, an average received $E_{s} / N_{0}$ of $30 \mathrm{~dB}$ is considered (Eq. 1). Similar conclusions are drawn nonetheless for lower moderate $E_{s} / N_{0}$ values.

We compare the performance of the SINR profile that maximizes throughput (blue plots with circle markers) with the uniform SINR profile that maximizes capacity [9] (red plots with asterisk markers). As the SINR profile that maximizes throughput is sensitive to the $\operatorname{PER}(\Gamma)$ function of the selected forward-error correcting (FEC) code, the performance is analyzed for two illustrative FEC codes (Fig. 1) : (a) a 3GPP based Turbo code of rate $1 / 3$ with $10 \%$ puncturing, as defined in [12]; (b) the classical convolutional code of rate $1 / 2$ and generator polynomials $133_{\text {oct }}$ and $171_{\text {oct }}$ (adopted in some standards such as DVB-S or IEEE 802.11). In both cases the packet length (information bits per packet) is $L=320$.

As shown in Fig. 1, the set of available PER values (asterisks) obtained by simulation are correctly fitted by means of a piecewise exponentially decreasing function as the following,

$$
\operatorname{PER}(\Gamma)=\left\{\begin{array}{cc}
\exp \left(-k_{1} \Gamma\right) & 0 \leq \Gamma \leq \Gamma_{t h} \\
\exp \left(k_{2}-k_{3} \Gamma\right) & \Gamma>\Gamma_{t h}
\end{array}\right.
$$

The constants $k_{1}, \Gamma_{t h}, k_{2}$ and $k_{3}$ for the studied FEC codes are: (a) for the 3GPP based Turbo code $k_{1}=0.05, \Gamma_{t h}=0.78$, $k_{2}=18.68$ and $k_{3}=24$; (b) for the convolutional code of rate $1 / 2 k_{1}=0.263, \Gamma_{t h}=1.35, k_{2}=6.77$ and $k_{3}=5.28$.

Fig. 2 depicts the system spectral efficiency versus the load $\alpha=K / N$ for the first studied FEC code (Turbo code). As shown, whereas the spectral efficiency for the uniform profile (red) degrades for large loads, the optimum profile (blue) maintains the maximum spectral efficiency. In Fig. 3,

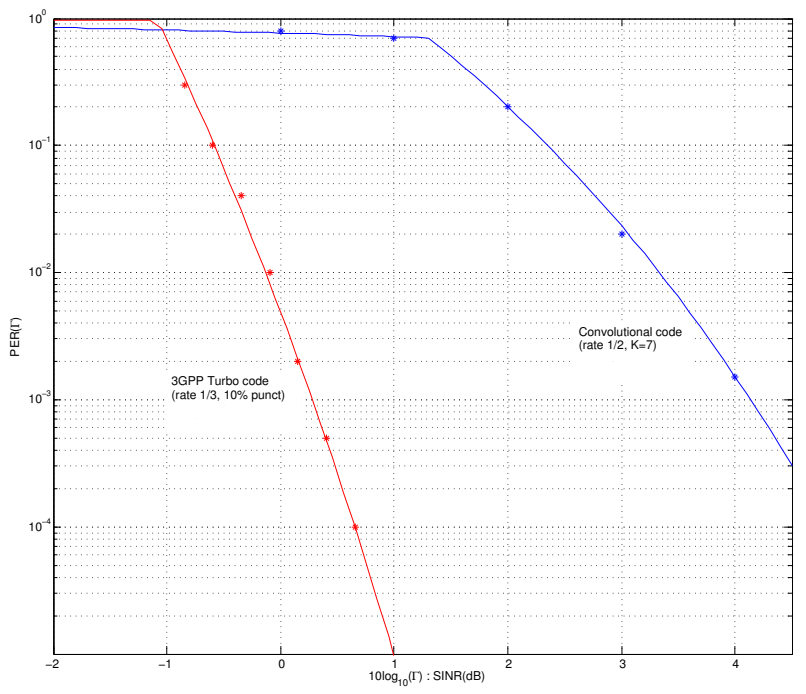

Fig. 1. PER vs. SINR curves for the two studied FEC codes: convolutional code (blue curve) and 3GPP Turbo code (red curve). Asterisks correspond to simulated points and continuous lines are the adopted piecewise exponentially-approximated curves. 


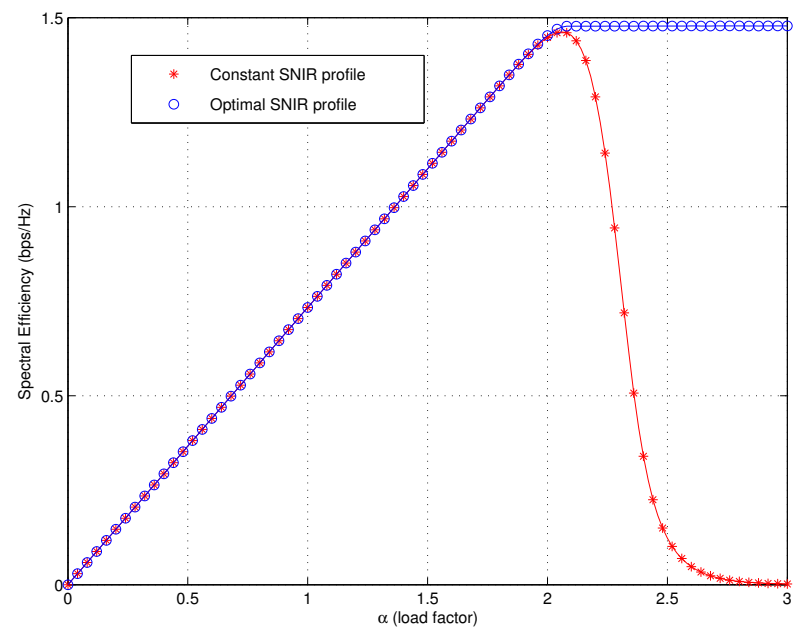

Fig. 2. Spectral Efficiency vs. traffic load $(\alpha)$ for the first studied FEC (Turbo code).
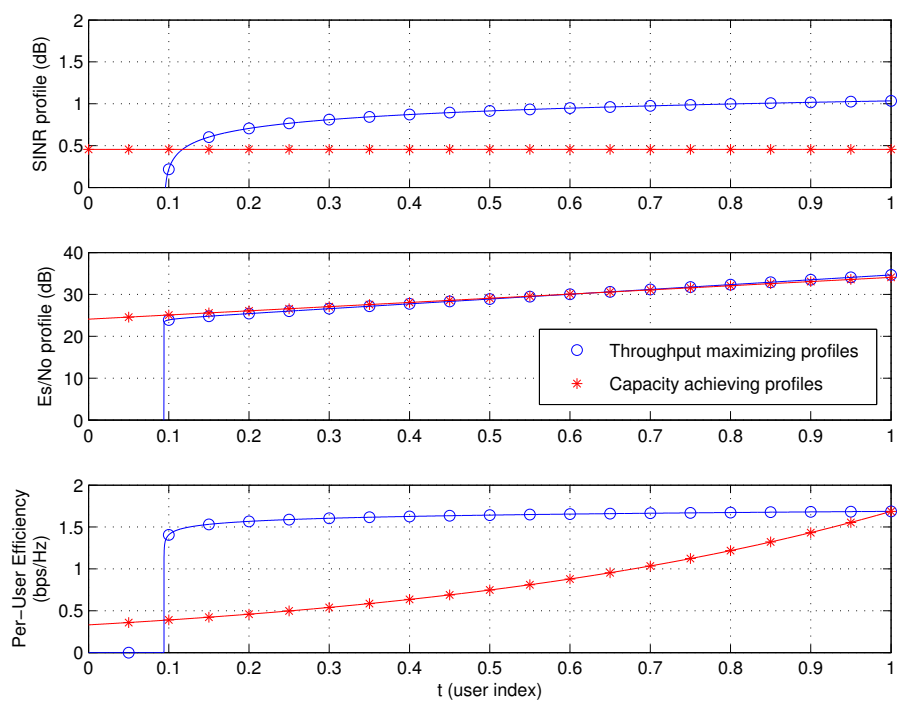

Fig. 3. Per-user profiles vs. user index $t$ for a $10 \%$ overloaded system ( $\alpha=2.30, K \approx 4710$ users) for the first studied FEC (Turbo code).

for the same FEC code, a $10 \%$ overloaded system $(\alpha=2.30)$ dealing with approximately $K=4710$ users is simulated. For this value of $\alpha$, the SINR profile $\Gamma(t)$ and the normalized $E_{s} / N_{0}$ profile $\dot{y}(t)$ are obtained using the theoretical analysis in Section III. The per-user efficiency profile is also calculated as the derivative of (12) with respect to the user index $t$,

$$
\dot{\mathrm{E}}(t)=\frac{\mathrm{d}}{\mathrm{d} t} \mathrm{E}(t)=\alpha R_{c} \exp \left(\alpha N \int_{t}^{1} f(\Gamma(v)) \mathrm{d} v\right)
$$

This simulation evidences that the SINR profile maximizing throughput (blue, circular markers) is not uniform in contrast to the SINR profile maximizing capacity (red, asterisks markers), which is constant for all the users [9].

The same curves are plotted in Fig. 4 and Fig. 5 for the second studied FEC (convolutional code). Since the convolutional code is less powerful than the studied Turbo code, the admissible load with this FEC is much lower than with the Turbo code (Fig. 4). In particular, the maximum accepted

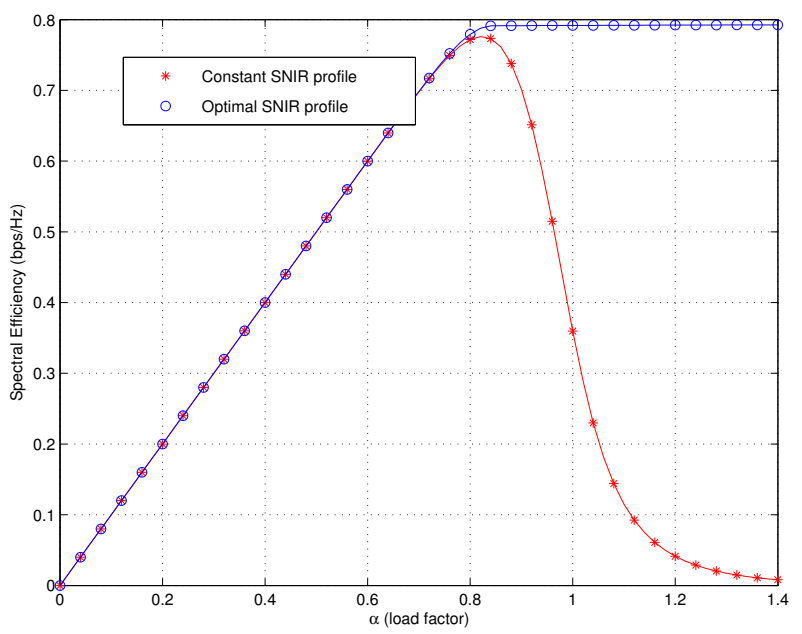

Fig. 4. Spectral Efficiency vs. traffic load $(\alpha)$ for the second studied FEC (convolutional code)
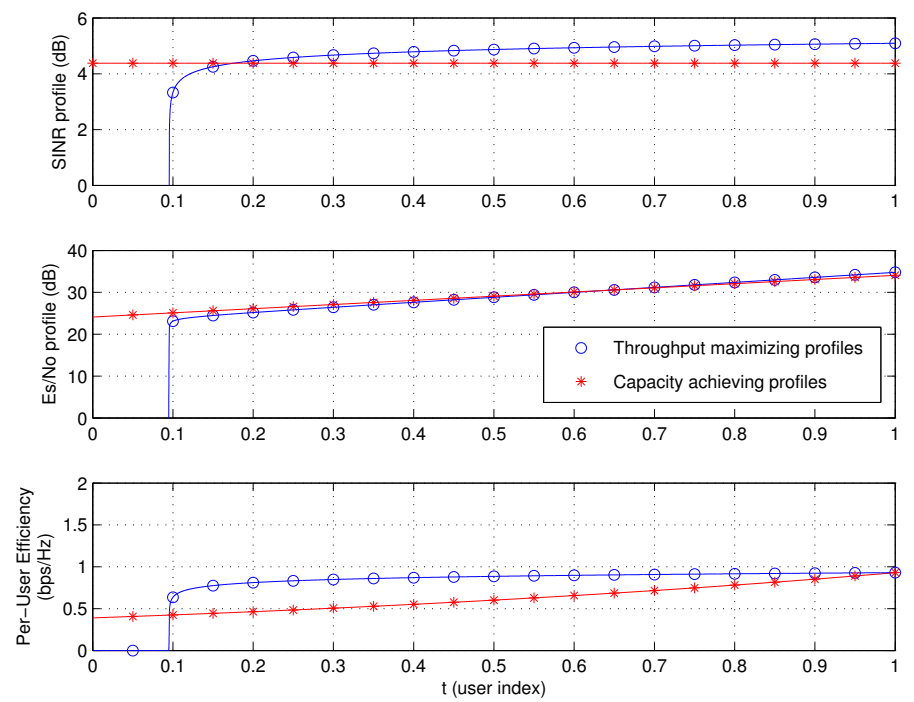

Fig. 5. Per-user profiles vs. user index $t$ for a $10 \%$ overloaded system $(\alpha=0.93, K \approx 1905$ users) for the second studied FEC (convolutional code).

load is approximately $\alpha=0.84$ (i.e. $K \approx 1720$ users). The optimum profiles in terms of throughput and capacity for the convolutional code appear depicted in Fig. 5 for comparison.

As is illustrated in Fig. 3 and Fig. 5, if the system is $10 \%$ overloaded, the $E_{s} / N_{0}$ profile maximizing throughput (blue, circles) assigns null power to $10 \%$ of the users in order to regulate the system load and maintain (maximize) the SIC performance in terms of spectral efficiency. This result is particularly relevant in the case of dynamic systems in which users select the transmitted power randomly on a frame-by-frame basis. In this setup, although users choose their transmitted power independently, they are able to induce collectively the $E_{s} / N_{0}$ profile that maximizes the overall system spectral efficiency, as explained in the next paragraph. Following this approach and using the proposed $E_{s} / N_{0}$ profile, the instantaneous load can be regulated in a distributed fashion in order to avoid the collapse of the system throughput in overloaded scenarios, as shown in Fig. 2 and Fig. 4. This 
allows the system to manage dynamically more users than the maximum number of users the capacity-achieving profile (red, asterisks) tolerates before the throughput drop-off.

An important advantage of the studied asymptotic (in the number of users) regime is that the $E_{s} / N_{0}$ profile $\gamma_{\text {opt }}(t)$ in (15) can be generated uncoordinatedly in some scenarios. This is possible because $\gamma_{\text {opt }}(t)$ only depends on public system parameters such as the spreading factor $N$, the total number of users $K$, the FEC characteristic curve or the average $E_{s} / N_{0}$ constraint $\bar{\gamma}$. The main idea is that users can use the $E_{s} / N_{0}$ profile for deciding, randomly, the transmitted symbol energy in every frame. In particular, in those scenarios in which the channel power gain $h_{k}^{2}$ is deterministic or near-deterministic, the transmitted symbol energy of user $k$ can be computed as $\left(N_{0} / h_{k}^{2}\right) \gamma_{\text {opt }}\left(t_{k}\right)$ where $t_{k}$ is a (independent) uniformly distributed random variable in the interval $[0,1]$.

\section{CONCLUSIONS}

We have shown, that in multiple access scenarios featuring a large number of users, it is feasible to express global performance (e.g., spectral efficiency) in terms of a limiting continuous distribution (or profile) for the symbol energy of the user population. This approach facilitates system optimization through application of Variational Calculus such that the optimum symbol energy profile and associated maximum performance can be determined by solving a differential equation with suitable boundary constraints. This framework is applicable to the whole range of system loads $K / N$ and predicts a seamless transition between an under- and an overloaded system. In the latter case, the obtained symbol energy distribution offers a natural way to regulate the load in the overloaded case. Notice, finally, that the analysis can be particularized to any FEC scheme in terms of its associated Packet Error Rate characteristic.

It is worth noting that the optimum $E_{s} / N_{0}$ and SINR profiles depend on the specific SIC policy implemented. Therefore, different results may be obtained when using other policies such as those described in [7], [14] (Multi-Branch SIC based on different user orderings) or [15] (Multi-Feedback SIC based on several candidates for cancellation).

\section{APPENDIX I. MODIFIED EULER-LAGRANGE EQUATION}

We seek to maximize the spectral efficiency $E$ in (8) with respect to $y(t)$ subject to the boundary constraints $y(0)=0$ and $y(1)=1$. Using the tools of Variational Calculus, the spectral efficiency can be expressed as a non-standard functional $\mathcal{G}(y)$ depending on the normalized aggregate $E_{s} / N_{0}$ profile $y(t)$, with $K=\alpha N$,

$$
\begin{aligned}
& \mathcal{G}(y)=\int_{0}^{1} \exp \left(K \int_{u}^{1} L(y, \dot{y} ; t) \mathrm{d} t\right) \mathrm{d} u \\
& L(y, \dot{y} ; t) \doteq f(\Gamma(t))=f\left(\frac{\dot{y}(t)}{a+b y(t)}\right),
\end{aligned}
$$

where, using the definition of the partial spectral efficiency $\mathrm{E}(t)$ in (12), we have $\mathcal{G}(y)=\frac{1}{\alpha R_{c}} \mathrm{E}(t)$. For $y_{\text {opt }}(t)$ the maximizing function, the standard procedure in Variational Calculus considers,

$$
y(t)=y_{\mathrm{opt}}(t)+\epsilon \cdot \eta(t),
$$

with $\eta(t)$ any function with $\eta(0)=\eta(1)=0$ (boundary constraints on $y(t)$ are not affected). Then, for $y_{\text {opt }}(t)$ to be a stationary point, we must have that $\nabla_{\epsilon=0}[\mathcal{G}(y)]=0$ for any such $\eta(t)$, which leads to a differential equation for $y(t)=y_{\text {opt }}(t)$, as will be shown. Hence, defining the auxiliary functional $\mathcal{M}(y ; u) \doteq K \int_{u}^{1} L(y, \dot{y} ; t) \mathrm{d} t$, we have,

$$
\begin{aligned}
\nabla_{\epsilon} \mathcal{G}(y) & =\int_{0}^{1} \exp [\mathcal{M}(y ; u)] \nabla_{\epsilon}[\mathcal{M}(y ; u)] \mathrm{d} u(21) \\
\nabla_{\epsilon}[\mathcal{M}(y ; u)] & =K \int_{u}^{1}\left(\partial_{y} L \cdot \nabla_{\epsilon} y+\partial_{\dot{y}} L \cdot \nabla_{\epsilon} \dot{y}\right) \mathrm{d} t(22)
\end{aligned}
$$

where, in (22), for shorter notation, the following partial derivatives are defined: $\partial_{y} L \doteq(\partial / \partial y) L(y, \dot{y} ; t)$ and $\partial_{\dot{y}} L \doteq$ $(\partial / \partial \dot{y}) L(y, \dot{y} ; t)$, as well as $y \doteq y(t)$ and $\dot{y} \doteq \dot{y}(t)$. From (20), we have that $\nabla_{\epsilon} y=\eta(t)$ and $\nabla_{\epsilon} \dot{y}=\dot{\eta}(t)=\nabla_{t} \eta(t)$. Hence, applying integration by parts to (22) and the boundary constraint $\eta(1)=0$, we get,

$$
\begin{aligned}
& \nabla_{\epsilon}[\mathcal{M}(y ; u)]= \\
& =K \int_{u}^{1}\left(\left[\partial_{y}-\nabla_{t} \partial_{\dot{y}}\right] L\right) \eta(t) \mathrm{d} t+\left.K\left(\left(\partial_{\dot{y}} L\right) \eta(t)\right)\right|_{u} ^{1} \\
& =K \int_{0}^{1}\left(\left[\partial_{y}-\nabla_{t} \partial_{\dot{y}}\right] L\right) \eta(t) \mathrm{u}(t-u) \mathrm{d} t-\left.K\left(\left(\partial_{\dot{y}} L\right) \eta(t)\right)\right|_{t=u}
\end{aligned}
$$

with $\mathrm{u}(t)$ the unit step function. For $\nabla_{\mathrm{EL}} \doteq \partial_{y}-\nabla_{t} \partial_{\dot{y}}$ the Euler-Lagrange diferentiation operator, we substitute (23) into (21), interchange integrals and set $\mathcal{G}^{\prime}=\nabla_{\epsilon} \mathcal{G}(y)$, so that,

$$
\begin{gathered}
\mathcal{G}^{\prime}=K \int_{0}^{1} \int_{0}^{1} \eta(t) \mathrm{u}(t-u) e^{\mathcal{M}(y ; u)}\left(\nabla_{\mathrm{EL}} L\right) \mathrm{d} t \mathrm{~d} u- \\
-\left.\int_{0}^{1} e^{\mathcal{M}(y ; u)} \eta(u)\left(\partial_{\dot{y}} L\right)\right|_{t=u} \mathrm{~d} u=\int_{0}^{1} \eta(t) w(t) \mathrm{d} t
\end{gathered}
$$

where, changing the integration variable from $u$ to $t$ in the second integral, $w(t)$ can be expressed as,

$$
w(t)=\int_{0}^{1} e^{\mathcal{M}(y ; u)} \mathrm{u}(t-u) \mathrm{d} u \cdot \nabla_{\mathrm{EL}} L-e^{\mathcal{M}(y ; t)} \partial_{\dot{y}} L
$$

From the Fundamental Lemma of Variational Calculus [13], we have that $\mathcal{G}^{\prime}=0$ for any $\eta(t)$ only if $w(t)=0$. Hence, defining the partial integral $\mathrm{A}(t)$,

$$
\mathrm{A}(t)=\int_{0}^{1} e^{\mathcal{M}(y ; u)} \mathrm{u}(t-u) \mathrm{d} u=\int_{0}^{t} e^{\mathcal{M}(y ; u)} \mathrm{d} u
$$

we have $\dot{\mathrm{A}}(t)=\nabla_{t} \mathrm{~A}(t)=\exp [\mathcal{M}(y ; t)]$ and, finally, setting $w(t)=0$ in (24), yields the modified Euler-Lagrange equation,

$$
\mathrm{A}(t) \cdot\left(\left[\partial_{y}-\nabla_{t} \partial_{\dot{y}}\right] L\right)=\dot{\mathrm{A}}(t) \cdot \nabla_{\dot{y}} L
$$

Now, we may multiply (26) by $\alpha R_{c}$ so that $\dot{\mathrm{E}}(t)=\alpha R_{c} \dot{\mathrm{A}}(t)$, where $\dot{\mathrm{E}}(t)=\nabla_{t} \mathrm{E}(t)=\alpha R_{c} \exp \left(K \int_{t}^{1} f(\Gamma(u)) d u\right)$ stands for the spectral efficiency density of user $t$, defined from the spectral efficiency $\mathrm{E}(t)$ in (12). Hence, $\mathrm{A}(t)$ may be substituted by $\mathrm{E}(t)$ in (26) and we can already proceed to derive the invariance equation in (11). Compacting notation : $\dot{\mathrm{E}} \doteq \dot{\mathrm{E}}(t)$ and $\Gamma \doteq \Gamma(t)$, and moving the negative term in (26) to the right-hand side, where $\mathrm{A}(t)=\mathrm{E}(t) /\left(\alpha R_{c}\right)$, we get,

$$
\begin{aligned}
\mathrm{E} \cdot \partial_{y} L & =\dot{\mathrm{E}} \cdot \partial_{\dot{y}} L+\mathrm{E} \cdot \nabla_{t} \partial_{\dot{y}} L \\
& =\nabla_{t}\left[\mathrm{E} \cdot \partial_{\dot{y}} L\right]
\end{aligned}
$$


As $\partial_{y} L=-f^{\prime}(\Gamma) \cdot b \dot{y} /(a+b y)^{2}=-b \Gamma \cdot \partial_{\dot{y}} L$ and $\partial_{\dot{y}} L=$ $f^{\prime}(\Gamma) /(a+b y)>0$ for $L=f(\dot{y} /(a+b y))$, (27) becomes,

$$
\begin{aligned}
-b \Gamma & =\nabla_{t}\left[\ln \left(\mathrm{E} \cdot \partial_{\dot{y}} L\right)\right] \\
& =\nabla_{t} \ln (\mathrm{E})+\nabla_{t} \ln \left(\partial_{\dot{y}} L\right)
\end{aligned}
$$

Operating on the last term in (28) and using (19) and (6), we get that $\nabla_{t} \ln \left(\partial_{\dot{y}} L\right)=\nabla_{t} \ln \left(f^{\prime}(\Gamma)\right)-b \Gamma$ with $f^{\prime}(\Gamma) \doteq$ $\nabla_{\Gamma} f(\Gamma)$ and, therefore, (28) can be written as,

$$
\begin{aligned}
& \nabla_{t} \ln (\mathrm{E})+\nabla_{t} \ln \left(f^{\prime}(\Gamma)\right)=0 \\
& \Rightarrow \quad \nabla_{t} \ln \left(\mathrm{E} \cdot f^{\prime}(\Gamma)\right)=0
\end{aligned}
$$

which, after integrating in $t$, yields (11), for some $c_{0}$ consistent with applicable boundary conditions, as is examined in appendix II when solving for the corresponding $\Gamma(t)$.

\section{APPENDIX II. DETERMINATION OF $\Gamma_{\text {opt }}(t ; c)$}

We may remove the two integrals in (12) by applying twice the operator $\nabla_{t}$ as follows,

$$
\nabla_{t} \ln \nabla_{t}\left[\frac{\mathrm{E}(t)}{\alpha R_{c}}\right]=-K f(\Gamma(t))
$$

Now, we substitute (11) into the left-hand side of (31). Hence, concealing the dependence on $t$ as $\Gamma \doteq \Gamma(t)$, we get,

$$
-K f(\Gamma)=\nabla_{t} \ln \nabla_{t}\left[\frac{c_{0} /\left(\alpha R_{c}\right)}{f^{\prime}(\Gamma)}\right]=\frac{\nabla_{t}^{2}\left[1 / f^{\prime}(\Gamma)\right]}{\nabla_{t}\left[1 / f^{\prime}(\Gamma)\right]}
$$

Now, let us define $\dot{\Gamma} \doteq \dot{\Gamma}(t) \doteq \nabla_{t} \Gamma(t)$ and use the following equality in (32), which contains the term $\nabla_{t}\left[1 / f^{\prime}(\Gamma)\right]$,

$$
\nabla_{t}\left[f(\Gamma) / f^{\prime}(\Gamma)\right]=\frac{f^{\prime}(\Gamma) \cdot \dot{\Gamma}}{f^{\prime}(\Gamma)}+f(\Gamma) \nabla_{t}\left[1 / f^{\prime}(\Gamma)\right]
$$

Solving for $f(\Gamma) \nabla_{t}\left[1 / f^{\prime}(\Gamma)\right]$ in (33) and subsituting into (32), we have that,

$$
\nabla_{t}^{2}\left[1 / f^{\prime}(\Gamma)\right]-K \dot{\Gamma}+K \nabla_{t}\left[f(\Gamma) / f^{\prime}(\Gamma)\right]=0
$$

Integration in $t$ may now be carried out from (34) to (35) to reduce by one the differential equation order,

$$
\nabla_{t}\left[1 / f^{\prime}(\Gamma)\right]-K \Gamma+K f(\Gamma) / f^{\prime}(\Gamma)=c,
$$

with $c$ a generic integration constant determined from the problem constraint specified at the end of this appendix. Calculating the derivative in $\nabla_{t}\left[1 / f^{\prime}(\Gamma)\right]$ and rearranging terms, we obtain the following first-order Ordinary Differential Equation (ODE),

$$
\dot{\Gamma}(t)=1 / \Psi_{c}(\Gamma(t))
$$

where $\Psi_{c}(\Gamma)$, which depends on the constant $c$, is given by

$$
\Psi_{c}(\Gamma) \doteq \frac{-f^{\prime \prime}(\Gamma)}{\left[f^{\prime}(\Gamma)\right]^{2}(K \mathrm{~h}(\Gamma)+c)}
$$

with $\mathrm{h}(\Gamma) \doteq \Gamma-f(\Gamma) / f^{\prime}(\Gamma)$ and $f^{\prime \prime}(\Gamma) \doteq \nabla_{\Gamma}^{2} f(\Gamma)$. In order to solve for $\Gamma(t)$ in (36), we use that $\dot{\Gamma}(t)=\mathrm{d} \Gamma / \mathrm{d} t$ is a ratio of differentials, which allows us to integrate in the continuous user index over the interval $[0, t]$, obtaining,

$$
\int_{0}^{\Gamma(t)} \Psi_{c}(\Gamma) \mathrm{d} \Gamma=\int_{0}^{t} \mathrm{~d} u=t
$$

where we have used $\Gamma(0)=0$, as concluded from (11) in Sec. II. Defining $\Omega_{c}(\Gamma) \doteq \int_{0}^{\Gamma} \Psi_{c}(x) \mathrm{d} x$, we get $\Omega_{c}(\Gamma(t))=t$ and, consequently, $\Omega_{c}(\Gamma)$ is the inverse function of $\Gamma(t)$ or, equivalently, the optimum SINR profile is expressed as,

$$
\Gamma_{\text {opt }}(t ; c)=\Omega_{c}^{-1}(t),
$$

in terms of $\Omega_{c}(\Gamma)$, which is calculated from the PER function of the adopted modulation/coding scheme for a given value of $c$. The value of $c$ in (37) may be selected in order to fulfil the average area constraint $\bar{\Gamma}_{0}$ in (14) while satisfying the positiveness of $\Psi_{c}(\Gamma)$ in (37), i.e., $c>-K \mathrm{~h}(\Gamma(1))$, which is indeed a necessary condition to guarantee that $\dot{y}(t) \geq 0$.

\section{ACKNOWLEDGMENT}

This work has been jointly supported by projects: (i) TEC2013-47020-C2-2-R (COMPASS), Ministerio de Economía y Comptetitividad, Spanish National Research Plan; (ii) 2014 SGR 60 AGAUR (Catalan government).

\section{REFERENCES}

[1] A. Zanella, M. Zorzi, A. F. dos Santos, P. Popovski, N. Pratas, C. Stefanovic, A. Dekorsy, C. Bockelmann, B. Busropan, T. A. H. J. Norp, "M2M massive wireless access: challenges, research issues, and ways forward", IEEE Globecom 2013, Atlanta, GA, USA, December 2013.

[2] E. Paolini, G. Liva, and M. Chiani, "High throughput random access via codes on graphs: Coded slotted ALOHA," IEEE Int. Conf. Commun. (ICC), Kyoto, Japan, Jun. 2011.

[3] O. Del Rio Herrero and R. De Gaudenzi, "High Efficiency Satellite Multiple Access Scheme for Machine-to-Machine Communications", IEEE Trans. Aerosp. Electron. Syst., vol. 48(4), pp. 2961-2989, Oct. 2012.

[4] E. Casini, R. De Gaudenzi and O. del Ro Herrero, "Contention resolution diversity slotted ALOHA (CRDSA): An enhanced random access scheme for satellite access packet networks.", IEEE Transactions on Wireless Communications, vol. 6(4), pp. 1408-1417, Apr. 2007.

[5] Y. Yu and G. B. Giannakis, "High-throughput random access using successive interference cancellation in a tree algorithm," IEEE Trans. Inf. Theory, vol. 53(12), pp. 4628-4639, Dec. 2007.

[6] A. Agrawal, J.G. Andrews, J.M. Cioffi, T.H. Meng, "Iterative power control for imperfect successive interference cancellation," IEEE Trans. on Wireless Comm., vol.4, no.3, May 2005.

[7] J. Sala, J. Villares, F. Rey, "Asymptotic and Finite-User PER Analysis of Successive Interference Cancellation for DS-CDMA", IEEE Comms. Letters, vol.15, no.11, pp. 1145-1147, November 2011.

[8] J.G. Andrews, T.H. Meng, "Optimum power control for successive interference cancellation with imperfect channel estimation", IEEE Trans. on Wireless Communications, vol.2(2), Mar 2003

[9] D. Djonin, V. K. Bhargava, "Asymptotic Analysis of the Conventional Decision Feedback Receiver in Fading Channels," IEEE Trans. on Wireless Commun., Sep. 2003.

[10] S. Verdu, S. Shamai, "Spectral Efficiency of CDMA with Random Spreading," IEEE Trans. on Information Theory, vol.45 (2), Mar. 1999.

[11] Y. Tadokoro, H. Okada, T. Yamazato, M. Katayama, "The optimum received signal-power distribution for CDMA packet communication systems employing successive interference cancellation" IEEE Int. Symposium on Spread Spectrum Techniques and Applications (ISSTA), pp. 37-41, Aug. 2004.

[12] "Simulation results of downlink puncturing algorithms", TSG-RAN Working Group 1(Radio) meeting \# 6, Espoo, Finland 13-16, July 1999.

[13] C. Ray Wilie, "Advanced Engineering Mathematics", 4th edition, McGraw-Hill, 1975.

[14] Rui Fa, R.C. de Lamare, "Design of adaptive multi-branch SIC receivers for MIMO spatial multiplexing systems", Sixth Int. Symposium on Wireless Communications Systems (ISWCS 2009), pp. 575-579.

[15] P. Li, R.C. de Lamare, R. Fa, "Multiple feedback successive interference cancellation detection for multiuser MIMO systems", IEEE Trans. on Wireless Communications, vol. 10, no. 8, pp. 2434-2439, August 2011. 